\title{
新型冠状病毒肺炎疫情期间 隔离人员心理状况调查研究
}

\author{
董人齐 ${ }^{1}$, 周 霞 $2^{2}$, 焦小楠 ${ }^{1}$, 郭炳杉 ${ }^{1}$, 孙立平 ${ }^{1}$, 王 芹 ${ }^{2}$ \\ 1 山东中医药大学, 山东济南 250014 ; \\ 2 山东中医药大学第二附属医院, 山东 济南 250001 \\ 通信作者:周霞,E-mail:lusy2000@126.com
}

收稿日期: 2020-02-21; 接受日期: 2020-02-23

DOI : 10.3724/SP.J.1329.2020.01004

开放科学 (资源服务) 标识码 (OSID) :

摘要目的: 探讨新型冠状病毒肺炎(COVID-19)疫情期间医学观察隔离人员心理因素变化特点, 为早期心 理指导、千预该群体提供依据。方法: 选取 40 例隔离人员,38例未隔离人员,采用广泛性焦虑量表(GAD-7) 以 及 PHQ-9 抑郁症篎查量表评价其心理状态。将 2 组心理因素进行对比分析。结果: 2 组在焦虑程度分级方 面有统计学意义 $(P<0.05)$, 抑郁程度分级差异无统计学意义 $(P>0.05)$; 在 “控制担忧” “难以放松” “无法静 坐” 3 项中, 差异无统计学意义 $(P>0.05)$; 但观察组其余各项指标均高于对照组, 差异有统计学意义 $(P<$ $0.05)$, 多项有显著统计学意义 $(P<0.01)$ 。2 组兴趣、睡眠、疲劳、食欲、专注力、行动力调查评分比较, 差异无 统计学意义 $(P>0.05)$; 但观察组在心情、挫败感、自伤意念的总评分显著高于对照组, 2 组比较差异有统计学 意义 $(P<0.05)$ 。结论: 隔离人员相比未隔离人员存在较多的负性情绪, 主要表现为对自身及家人身体状况不 确定的焦虑、紧张情绪。

关键词新型冠状病毒肺炎; 医学隔离; 焦虑; 抑郁

自 2019 年 12 月以来, 新型冠状病毒肺炎 (Corona Virus Disease 2019,COVID-19)在全国乃至世界 范围内陆续扩散, 因其突发性、传染性, 以及“人传 人”的传播方式 ${ }^{[1]}$, 对社会经济、教育等产生了巨大 影响。由于早期对疾病风险不确定、防护措施不到 位等情况, 造成了当前疾病曼延, 已对大量医护人 员产生了不同程度的心理影响 ${ }^{[2-3]}$ 。依据《中华人民 共和国传染病防治法》,COVID-19 已被国家纳人乙类 传染病范围, 采取甲类传染病的预防、控制措施 ${ }^{[4-5]}$ 。 各地均采取 “确诊病人隔离治疗、密切接触者隔离 医学观察、普通人居家隔离” 等一系列防控措施。为 了解这一特殊时期不同环境下 (是否与确诊病例直 接或间接接触)群众的身心状况, 特开展此项问卷 调查, 为早期指导社会群体身心健康提供依据。

\section{1 资料与方法}

\section{1 一般资料}

选取 2020 年 2 月 6 日与确诊患者有直接或间 接接触的医学观察隔离人员 40 例作为观察组, 并 以同期省内未隔离人员 38 名作为对照组。2 组居民 年龄、性别及受教育程度等一般资料比较差异均无 统计学意义 $(P>0.05)$, 具有可比性, 见表 1 。

1.2 方法

1.2.1 调查工具 自制问卷, 收集一般资料, 包括 性别、年龄段、学历和对 COVID-19 的了解情况。采 用信效度较好的广泛性焦虑量表 (Generalized Anxiety Scale,GAD-7 $)^{[6]}$ 和 PHQ-9 抑郁症篮查量表, 本 调查用来篮查、评估居民焦虑、抑郁状况。(1) GAD-7

引用格式:董人齐,周霞,焦小楠,等. 新型冠状病毒肺炎疫情期间隔离人员心理状况调查研究 $[\mathrm{J}]$. 康复学报, 2020,30(1):7-10.

DONG R Q,ZHOU X, JIAO X N, et al. Psychological status in medical isolation persons during outbreak of COVID-19 [J]. Rehabilitation Medicine,2020,30

(1) $: 5-8$.

DOI : $10.3724 /$ SP.J.1329.2020.01004 
表 12 组一般资料比较 $(n, \%)$

Table 1 Comparison of general data between two groups $(n, \%)$

\begin{tabular}{|c|c|c|c|c|c|c|c|c|c|}
\hline \multirow{2}{*}{ 组 别 } & \multirow{2}{*}{ 例数 } & \multicolumn{2}{|c|}{ 性别 } & \multicolumn{3}{|c|}{ 文化程度 } & \multicolumn{3}{|c|}{ 年龄/岁 } \\
\hline & & 男 & 女 & 高中 & 大学 & 硕士及以上 & $21 \sim 30$ & $31 \sim 40$ & $41 \sim 50$ \\
\hline 对照组 & 38 & $16(42.11)$ & $22(57.89)$ & $4(10.53)$ & $18(47.37)$ & $16(42.11)$ & $12(31.58)$ & $20(52.63)$ & $6(15.79)$ \\
\hline 观察组 & 40 & $17(42.50)$ & $23(57.50)$ & $1(2.50)$ & $27(67.50)$ & $12(30.00)$ & $7(17.50)$ & $25(62.50)$ & $8(20.00)$ \\
\hline
\end{tabular}

由 7 个条目组成, 以了解测试者在过去 2 周内有多少 时间受到包括 7 个方面的困扰。每项的评分范围均 是 $0 \sim 3$ 分, 分别代表的时间频率如下: 完全没有、偶 尔几天有、经常有、几乎每天, 累加各项分值作为总 分。评分以 $5 、 10 、 15$ 分判定轻度、中度、重度焦虑程 度分界值。(2) PHQ-9 量表涵盖 9 个条目,无需专业 人员进行评价, 具有简便、高效的特点 ${ }^{[7]}$, 是基层医 疗诊所评估抑郁症的最常用工具 ${ }^{[8-9]}$ 。主要包括 9 个 症状因子, 分别是兴趣、心情、睡眠、疲劳、食欲、挫 败感、专注力、行动力、躯体伤害倾向。每项的评分 范围均为 $0 \sim 3$ 分, 赋分原则同上。累加各项分值算 得总分, 以 $5 、 10 、 15 、 20$ 分划分轻度、中度、中重度、 重度抑郁程度等级。其中第 $1 、 4$ 项, 代表抑郁的核心 症状,第 9 项提示测试者有自伤意念, 此 3 项任意 一项得分超过 1 分,应予以关注。

1.2.2 调查方法 本次调查为避免增加患者心理 负担, 采用匿名问卷调查方式。研究者将问卷录人 “问卷星”调查程序, 经过预调查完善相关条目。通 过物业管理人员加人相应小区微信群, 向所有成员 介绍此调查的目的和意义后发布问卷, 人组人员均 自愿扫码填写完成量表。

1.2.3 质量控制 本次调查采用网络问卷方式, 相 同 IP 地址只能作答 1 次, 保证填写人为本人。问卷
全部填写完毕方可提交,同时实时进行后台监控, 以保证数据可靠性。本次调查共发放问卷 84 份, 评 定调查结果, 排除不合格问卷, 如可明显看出规律 性的答案、前后逻辑不通者, 共收回有效问卷 78 份, 问卷有效率为 $92.86 \%$ 。

\section{3 统计学方法}

采用 SPSS 21.0 统计软件进行数据分析。计量资 料服从正态分布以 $(\bar{x} \pm s)$ 表示, 组间比较采用 $t$ 检验, 否则, 采取 Mann-Whitney 秩和检验, 以 $95 \%$ 置信区 间 $\left(P_{2.5} \sim P_{97.5}\right)$ 表示; 计数资料用例 $(\%)$ 表示, 采用 $\chi^{2}$ 检验。以 $P<0.05$ 为差异有统计学意义。

\section{2 结 果}

2 组 GAD-7 量表及 PHQ-9 抑郁症篎查量表结 果不符合正态分布, 故采用 Mann-Whitney 秩和检 验进行比较。2 组达到焦虑、抑郁程度诊断标准的分 值符合正态分布, 组间比较采用独立样本 $t$ 检验。

\subsection{2 组 GAD-7 量表调查结果}

2 组在“不能够停止或控制担忧、很难放松下来、 由于不安而无法静坐”3 项中, 差异无统计学意义 $(P>0.05)$; 但观察组其余因子评分均高于对照组, 差异有统计学意义 $(P<0.05)$, 多项有显著统计学意 义 $(P<0.01)$ 。见表 2 。

表 22 组 GAD-7 量表得分比较 $\left(\boldsymbol{P}_{2.5} \sim \boldsymbol{P}_{97.5}\right)$

Table 2 Comparison of GAD-7 scores between two groups $\left(\boldsymbol{P}_{2.5} \sim \boldsymbol{P}_{97.5}\right)$

\begin{tabular}{|c|c|c|c|c|c|}
\hline 组 别 & $n$ & 感觉紧张、焦虑或急切 & 不能够停止或控制担忧 & 对各种各样的事情担忧过多 & 很难放松下来 \\
\hline 对照组 & 38 & $0.3678 \sim 0.7901$ & $0.3138 \sim 0.7389$ & $0.2650 \sim 0.6298$ & $0.2906 \sim 0.6567$ \\
\hline 观察组 & 40 & $0.7894 \sim 1.3106$ & $0.5191 \sim 0.1009$ & $0.6733 \sim 1.1267$ & $0.4306 \sim 0.9194$ \\
\hline$Z$ 值 & & -2.758 & -1.422 & -2.947 & -1.025 \\
\hline$P$ 值 & & $<0.01$ & $>0.05$ & $<0.01$ & $>0.05$ \\
\hline 组 别 & $n$ & 由于不安而无法静坐 & 变得容易烦恼或急躁 & 感到似乎将有可怕的事情发生而害怕 & 总分 \\
\hline 对照组 & 38 & $0.1165 \sim 0.4098$ & $0.2149 \sim 0.5746$ & $0.2566 \sim 0.5855$ & $2.1794 \sim 4.0311$ \\
\hline 观察组 & 40 & $0.2256 \sim 0.5744$ & $0.5197 \sim 0.8803$ & $0.5640 \sim 0.9860$ & $4.0872 \sim 6.4628$ \\
\hline$Z$ 值 & & -1.110 & -2.437 & -2.400 & -2.584 \\
\hline$P$ 值 & & $>0.05$ & $<0.05$ & $<0.01$ & $<0.01$ \\
\hline
\end{tabular}




\subsection{2 组 PHQ-9 抑郁症篮查量表调查结果}

2 组兴趣、睡眠、疲劳、食欲、专注力、行动力调 查评分比较, 差异无统计学意义 $(P>0.05)$; 但观察 组在心情、挫败感、自伤意念的评分显著高于对照
组, 2 组比较差异有统计学意义 $(P<0.05)$, 尤其心 情低落、挫败感等心理因子比较差异具有显著统计 学意义 $(P<0.01)$ 。见表 3 。

表 32 组 PHQ-9 量表得分比较 $\left(\boldsymbol{P}_{2.5} \sim \boldsymbol{P}_{97.5}\right)$

Table 3 Comparison of PHQ-9 scores between two groups $\left(\boldsymbol{P}_{2.5} \sim \boldsymbol{P}_{97.5}\right)$

\begin{tabular}{|c|c|c|c|c|c|c|c|}
\hline 组 别 & $n$ & $\begin{array}{l}\text { 做事时提不起 } \\
\text { 劲或没有兴趣 }\end{array}$ & $\begin{array}{l}\text { 感到心情低落、 } \\
\text { 沮丧或绝望 }\end{array}$ & $\begin{array}{l}\text { 人睡困难、睡不 } \\
\text { 安或睡得过多 }\end{array}$ & $\begin{array}{l}\text { 感觉疲倦或 } \\
\text { 没有活力 }\end{array}$ & $\begin{array}{l}\text { 食欲不振 } \\
\text { 或吃太多 }\end{array}$ & $\begin{array}{l}\text { 觉得自己很糟或觉得自己很 } \\
\text { 失败,或让自己、家人失望 }\end{array}$ \\
\hline 对照组 & 38 & $0.3147 \sim 0.7960$ & $0.1905 \sim 0.5463$ & $0.2353 \sim 0.6594$ & $0.3406 \sim 0.7647$ & $0.0747 \sim 0.3463$ & $0.0030 \sim 0.2075$ \\
\hline 观察组 & 40 & $0.4541 \sim 0.9959$ & $0.5358 \sim 0.9642$ & $0.3977 \sim 0.8023$ & $0.4377 \sim 0.8123$ & $0.1877 \sim 0.5623$ & $0.2023 \sim 0.5477$ \\
\hline$Z$ 值 & & -0.870 & -2.633 & -1.228 & -0.678 & -1.231 & -2.570 \\
\hline$P$ 值 & & $>0.05$ & $<0.01$ & $>0.05$ & $>0.05$ & $>0.05$ & $<0.01$ \\
\hline
\end{tabular}

\begin{tabular}{|c|c|c|c|c|c|}
\hline 组 别 & $n$ & $\begin{array}{l}\text { 对事物专注有困难,例 } \\
\text { 如看报纸或看电视时 }\end{array}$ & $\begin{array}{l}\text { 行动或说话速度缓慢到别人已经察觉? 或刚好相 } \\
\text { 反一一变得比平日更烦躁或坐立不安, 动来动去 }\end{array}$ & $\begin{array}{l}\text { 有不如死掉或用某种 } \\
\text { 方式伤害自己的念头 }\end{array}$ & 总分 \\
\hline 对照组 & 38 & $0.0886 \sim 0.4903$ & $0.0190 \sim 0.2442$ & $0.0270 \sim 0.0796$ & $1.6530 \sim 3.7155$ \\
\hline 观察组 & 40 & $0.2091 \sim 0.6409$ & $0.1348 \sim 0.4652$ & $0.0519 \sim 0.2981$ & $3.0903 \sim 5.6097$ \\
\hline$Z$ 值 & & -1.100 & -1.591 & -2.150 & -2.114 \\
\hline$P$ 值 & & $>0.05$ & $>0.05$ & $<0.05$ & $<0.05$ \\
\hline
\end{tabular}

\subsection{2 组焦虑、抑郁程度比较}

统计 2 组居民量表总分发现,观察组达到焦虑、 抑郁的人数和程度均明显高于对照组,焦虑合并抑 郁者明显多于对照组，出现负性情绪的居民占 $57.50 \%$, 明显高于对照组的 $34.21 \%$ 。根据 GAD-7 量表结果
分析, 隔离人员与未隔离人员相比, 焦虑程度更明 显, 2 组比较差异有统计学意义 $(P<0.05)$ 。尽管隔 离居民抑郁发生率高于普通居民, 但 2 组比较差异 无统计学意义 $(P>0.05)$, 考虑与样本量小有关。见 表 4 和表 5 。

表 42 组焦虑、抑郁程度比较 $(n, \%)$

Table 4 Comparison of degree of subject's anxiety and depression between two groups $(n, \%)$

\begin{tabular}{|c|c|c|c|c|c|c|c|c|c|c|c|c|}
\hline \multirow{2}{*}{ 组 别 } & \multicolumn{4}{|c|}{ GAD-7(焦虑) } & \multicolumn{5}{|c|}{ PHQ-9(抑郁) } & \multicolumn{3}{|c|}{ 焦虑/抑郁 } \\
\hline & 轻度 & 中度 & 重度 & 无 & 轻度 & 中度 & 中重度 & 重度 & 无 & 焦虑合并抑郁 & 单一 & 二者均无 \\
\hline 对照组 & $12(31.58)$ & $0(0)$ & $0(0)$ & $26(68.42)$ & $6(15.79)$ & $1(2.63)$ & $0(0)$ & $0(0)$ & $31(81.58)$ & $6(15.79)$ & $7(18.42)$ & $25(65.79)$ \\
\hline 观察组 & $17(42.50)$ & $6(15.00)$ & $0(0)$ & $17(42.50)$ & $12(30.00)$ & $3(7.50)$ & $1(2.50)$ & $0(0)$ & $24(60.00)$ & $13(32.50)$ & $10(25.00)$ & $17(42.50)$ \\
\hline
\end{tabular}

表 52 组焦虑、抑郁程度比较 $(\bar{x} \pm s)$

Table 5 Comparison of degree of subject's anxiety and depression between two groups $(\bar{x} \pm s)$

\begin{tabular}{cccc}
\hline 组 别 & $n$ & 焦虑 & 抑郁 \\
\hline 对照组 & 38 & $3.17 \pm 2.84$ & $9.00 \pm 0.77$ \\
观察组 & 40 & $5.33 \pm 3.74$ & $8.22 \pm 0.74$ \\
$t$ 值 & & -2.207 & -0.614 \\
$P$ 值 & & $<0.05$ & $>0.05$ \\
\hline
\end{tabular}

\section{3 讨 论}

本研究通过对 40 例接受医学观察隔离人员及 同期 38 例未隔离人员的心理健康状况进行了横断 面流行病学调查,发现接受医学观察隔离人员一般 健康状况与未隔离人员无明显差异, 2 组均有一些
负性情绪, 这与本次疫情波及面广, 大家都处于不 同的隔离状态下有关。对于突如其来的疫情, 人们 一时出现情绪变化是疫情特殊环境下的正常心理 情绪反应。接受医学观察隔离组在焦虑、抑郁程度 分级明显高于对照组, 与所处的环境有关, 且有少 数居民出现 “自伤、自杀意念”,处于心理应激状态。 经过心理援助专家一对一的应急援助, 通过应用运 用本土化的中医心理 “移空技术” (疫情版) 减少恐 惧, 改善身心症状, 中医功法 “八段锦” 练习进行居 家健身锻炼的中医综合身心技术, 引导其释放不良 情绪, 增强内在力量及自我稳定性, 传授正确的疫情 相关防护知识, 建立安全感。并和社区工作人员联 系, 加强观察及生活支持, 以防止进一步发展为自 杀、自伤计划或行为, 为相关居民提供了恰当的社 
会心理支持,经危机干预后居民情绪稳定。

医学观察隔离人员负面情绪形成原因分析: (1) 担心感染。该群体均与确诊患者有直接或间接接触, 担心自己或家人感染病毒。(2)生活无序。原本生活 状态被打乱, 规划无法实施, 加剧了个体的精神压力 和不适感, 致使心理健康水平显著下降。(3) 环境因 素刺激。隔离期间小区内部处于较为紧张的状态, 隔 离人员长时间处在同一空间里, 容易产生视觉疲劳、 憋闷感等生理不适。(4) 网络與论导向。信息传递速 度更快, 很多信息难辨真伪, 对外界情况的不了解, 加重了人们的猜疑和担心, 容易引起恐惧心理。

COVID-19 疫情会给人们带来巨大的心理压 力, 并引发较多复杂的社会心理问题。因此, 在目前 较长时间居家隔离环境下, 社会各部门及医疗卫生 界需要认识到维护心理健康的重要性, 有责任采取 有效、快捷、简便的措施, 识别特殊群体、个体焦虑、 抑郁障碍的发生并及时采取防护措施。关于心理援 助在应激状态的急救方面, 不仅能教授大众一些易 于操作的身心稳定化技术如 “蝴蝶拍” “三调放松” 等, 还可以提供陪伴、倾听与疫情相关科普知识等 支持, 使隔离人员缓解焦虑、恐慌状态, 促进其情绪 稳定。在 “全民抗疫” 关键时刻, 倡议大家提高心理 情绪的自我觉察力, 及时发现恐慌、焦虑等“心理病 毒”, 积极寻求帮助, 及早接受心理辅导或应急援助。 同时学会自我调节, 寻找生活中的“小欢喜”, 使心 理疏导和心理干预发挥出最佳效能。

\section{参考文献}

[1] 中国疾病预防控制中心新型冠状病毒感染的肺炎疫情一级 响应态势分析与风险评估组. 2019 新型冠状病毒疫情进展 和风险评估 $[\mathrm{R} / \mathrm{OL}]$. (2020-01-28) [2020-01-29]. http :// news.medlive.cn/pul/info-progress/show-165643_145.html.

[2] 徐明川, 张悦. 首批抗击新型冠状病毒感染肺炎的临床一线支 援护士的心理状况调查 [J]. 护理研究,2020,34(3):368-370.

[3] 梅俊华, 张琦, 龚雪, 等. 新型冠状病毒肺炎感染医护人员心 理及睡眠状态分析 [J/OL]. 医药导报. (2020-02-17) [2020$02-21$ ]. http ://kns.cnki.net/kcms/detail/42.1293.R.20200215. 0150.002.html.

[4] ZHU N,ZHANG D, WANG W, et al. A novel coronavirus from patients with pneumonia in China,2019 [J]. N Engl J Med,2020, $382(8): 727-733$.

[5] 湖北省医学会儿科学分会, 武汉医学会儿科学分会, 湖北省 儿科医疗质量控制中心. 湖北省儿童新型冠状病毒感染诊疗 建议 (试行第一版) [J]. 中国当代儿科杂志, 2020,22(2): 96-99.

[6]王瑜, 陈然, 张岗. 广泛性焦虑量表-7在中国综合医院住院 患者中的信效度研究 $[J]$. 临床精神医学杂志, 2018,28(3): 168-171.

[7] 秦泽慧, 梁列新. 中文版 PHQ-9 在不同人群篮查抑郁症的最 佳截止值的研究分析 [J]. 临床消化病杂志, 2019,31(5):333336.

[8] DARAY F M, HUNTER F, ANASTASIA A, et al. Psychometric properties of the Patient Health Questionnaire nine-item version (PHQ-9) for use among hospitalized non-psychiatric medical patients [J]. Gen Hosp Psychiatry, 2019,61:69-75.

[9] MITCHELL H G, FRAYNE D, WYATT B, et al. Comparing the PHQ -9 to the multidimensional behavioral health screen in predicting depression-related symptomatology in a primary medical care sample [J]. J Pers Assess, 2020,102(2):175-182.

\title{
Psychological Status in Medical Isolation Persons during Outbreak of COVID-19
}

DONG Renqi ${ }^{1}$, ZHOU Xia ${ }^{2 *}$, JIAO Xiaonan ${ }^{1}$, GUO Bingshan ${ }^{1}$, SUN Liping ${ }^{1}$, WANG Qin ${ }^{2}$

${ }^{1}$ Shandong University of Traditional Chinese Medicine, Jinan, Shandong 250014, China;

${ }^{2}$ The Second Affiliated Hospital, Shandong University of Traditional Chinese Medicine, Jinan, Shandong 250001, China

*Correspondence: ZHOU Xia, E-mail: lusy2000@126.com

\begin{abstract}
Objective: To explore the psychological change factors in quarantined personnel during the outbreak of Corona Virus Disease 2019 (COVID-19), and to provide basis for the early psychological guidance and intervention. Methods: A total of forty isolated individuals and 38 unisolated individuals were selected, and their general mental anxiety was evaluated by the Generalized Anxiety Scale (GAD-7) and PHQ-9 Depression Screening Scale. The psychological factors of the two groups were compared and analyzed. Results: There was statistical significance in the level of anxiety $(P<0.05)$, and no significant difference in the level of depression $(P>0.05)$ between the two groups. There were no significant differences in the three items of "worrying control", "relaxing difficulty", and "unable to sit still" $(P>0.05)$; however, the other indicators in the observation group were higher than those in the control group, and the differences were statistically significant $(P<0.05)$, and most of them were very statistically significant $(P<0.01)$. There were no significant differences in the scores of the two groups on interest, sleep, fatigue, appetite, concentration, and mobility ( $P>0.05)$; but the overall scores of mood, frustration, and self-injury in the observation group were significantly higher than those in the control group, there were statistically significant differences between the two groups $(P<0.05)$. Conclusion: Compared with un-isolated individuals, the isolated individuals have more negative emotions, which are mainly manifested as anxiety and nervousness about the physical condition of themselves and their families.
\end{abstract}

KEY WORDS Corona Virus Disease 2019; medical isolation; anxiety; depression

DOI : 10.3724/SP.J.1329.2020.01004 